\title{
Evaluation of urinary resveratrol as a biomarker of dietary resveratrol intake in the European Prospective Investigation into Cancer and Nutrition (EPIC) study
}

Raul Zamora-Ros ${ }^{1 *}$, Joseph A. Rothwell ${ }^{2}$, David Achaintre ${ }^{2}$, Pietro Ferrari ${ }^{2}$, Marie-Christine BoutronRuault $^{3,4}$, Francesca R. Mancini ${ }^{3,4}$, Aurelie Affret ${ }^{3,4}$, Tilman Kühn ${ }^{5}$, Verena Katzke ${ }^{5}$, Heiner Boeing ${ }^{6}$, Sven Küppel ${ }^{6}$, Antonia Trichopoulou ${ }^{7,8}$, Pagona Lagiou ${ }^{7,8,9}$, Carlo La Vecchia ${ }^{7,10}$, Domenico Palli ${ }^{11}$, Paolo Contiero ${ }^{12}$, Salvatore Panico ${ }^{13}$, Rosario Tumino ${ }^{14}$, Fulvio Ricceri ${ }^{15,16}$, Hwayoung Noh $^{2}$, Heinz Freisling ${ }^{2}$, Isabelle Romieu ${ }^{2}$ and Augustin Scalbert ${ }^{2}$

${ }^{1}$ Unit of Nutrition and Cancer, Epidemiology Research Program Catalan Institute of Oncology, Bellvitge Biomedical Research Institute (IDIBELL), 08908 Barcelona, Spain

${ }^{2}$ Section of Nutrition and Metabolism, International Agency for Research on Cancer (IARC-WHO), 69372 Lyon, France ${ }^{3}$ Centre de recherche en épidémiologie et santé des populations (CESP), Institut National de la Santé et de la Recherche Médicale (INSERM), Université de Versailles Saint-Quentin-en-Yvelines (UVSQ), Université Paris-Sud, Université Paris-Saclay, 94805 Villejuif, France

${ }^{4}$ Gustave Roussy Institute, 94805 Villejuif, France

${ }^{5}$ Division of Cancer Epidemiology, German Cancer Research Center, 69120 Heidelberg, Germany

${ }^{6}$ Department of Epidemiology, German Institute of Human Nutrition Potsdam-Rehbruecke, 14558 Nuthetal, Germany

${ }^{7}$ Hellenic Health Foundation, GR-115 27, Athens, Greece

${ }^{8}$ Unit of Nutritional Epidemiology and Nutrition in Public Health, Department of Hygiene, Epidemiology and Medical Statistics, School of Medicine, WHO Collaborating Center for Nutrition and Health, National and Kapodistrian University of Athens, 10679 Athens, Greece

${ }^{9}$ Department of Epidemiology, Harvard T.H. Chan School of Public Health, Boston, MA 02115, USA

${ }^{10}$ Department of Clinical Sciences and Community Health, Università degli Studi di Milano, 20122 Milan, Italy

${ }^{11}$ Cancer Risk Factors and Life-Style Epidemiology Unit, Cancer Research and Prevention Institute (ISPO), 50141 Florence, Italy

${ }^{12}$ Environmental Epidemiology Unit, Fondazione IRCCS (Istituto di Ricovero e Cura a Carattere Scientifico) Istituto Nazionale dei Tumori, 20133 Milan, Italy

${ }^{13}$ Dipartimento di Medicina Clinica e Chirurgia, Federico II University, 80131 Naples, Italy

${ }^{14}$ Cancer Registry and Histopathology Unit, 'Civic M.P. Arezzo' Hospital, 97100 Ragusa, Italy

${ }^{15}$ Unit of Epidemiology, Regional Health Service ASL TO3, Grugliasco (TO), 10093 Turin, Italy

${ }^{16}$ Department of Clinical and Biological Sciences, University of Turin, 10043 Turin, Italy

(Submitted 21 February 2017 - Final revision received 4 May 2017 - Accepted 17 May 2017- First published online 22 June 2017)

\section{Abstract}

In vitro studies have shown several beneficial properties of resveratrol. Epidemiological evidence is still scarce, probably because of the difficulty in estimating resveratrol exposure accurately. The current study aimed to assess the relationships between acute and habitual dietary resveratrol and wine intake and urinary resveratrol excretion in a European population. A stratified random subsample of 475 men and women from four countries participating in the European Prospective Investigation into Cancer and Nutrition (EPIC) cross-sectional study, who had provided 24-h urine samples and completed a 24-h dietary recall (24-HDR) on the same day, were included. Acute and habitual dietary data were collected using standardised 24-HDR software and a validated country-specific dietary questionnaire, respectively. PhenolExplorer was used to estimate the intake of resveratrol and other stilbenes. Urinary resveratrol was analysed using tandem MS. Spearman's correlation coefficients between estimated dietary intakes of resveratrol and other stilbenes and consumption of wine, their main food source, were very high $(r>0.9)$ when measured using dietary questionnaires and were slightly lower with 24-HDR $(r>0 \cdot 8)$. Partial Spearman's

Abbreviations: 24-HDR, 24-h dietary recall; DQ, dietary questionnaire.

* Corresponding author: Dr R. Zamora-Ros, email rzamora@iconcologia.net 
correlations between urinary resveratrol excretion and intake of resveratrol, total stilbenes or wine were found to be higher when using the 24-HDR ( $R_{\text {partial }}^{2}$ approximately 0.6) than when using the dietary questionnaires $\left(R_{\text {partial }}^{2}\right.$ approximately $\left.0 \cdot 5\right)$. Moderate to high correlations between dietary resveratrol, total stilbenes and wine, and urinary resveratrol concentrations were observed. These support the earlier findings that 24-h urinary resveratrol is an effective biomarker of both resveratrol and wine intakes. These correlations also support the validity of the estimation of resveratrol intake using the dietary questionnaire and Phenol-Explorer.

Key words: Resveratrol: Biomarkers: Urine: Intakes: Wine

Resveratrol (3,5,4'-trihydroxystilbene) is the most well-known stilbene. This polyphenol class also includes piceid (resveratrol glucoside), pallidol and viniferins (resveratrol oligomers), and piceatannol (tetrahydroxystilbene). In the last two decades, resveratrol and other stilbenes have received considerable attention, particularly in in vitro and preclinical studies, because of their various biological properties such as antioxidant and anti-inflammatory effects ${ }^{(1,2)}$. Their capacity to modulate several signalling pathways leads to important cellular processes such as apoptosis, inhibition of angiogenesis and tumour growth as well as lipidic and glycaemic modulation ${ }^{(1,2)}$. However, evidence from epidemiological studies regarding effects on health is lacking, because of the difficulty in accurately estimating resveratrol exposure in large population-based samples, and because of the low levels of resveratrol observed in humans in non-experimental conditions compared with those in experimental conditions ${ }^{(2,3)}$.

Resveratrol and its derivatives are found in at least seventy-two plant species, including twelve families and thirty-one genera, but they are found in only a limited number of edible foods (online Supplementary Table S1) ${ }^{(4,5)}$. The primary sources of stilbenes are grapes and wine, especially red wine ${ }^{(4,5)}$. They can also be found in minor concentrations in peanuts, pistachios, berries $^{(4,5)}$, dark chocolate ${ }^{(6)}$, some beers ${ }^{(7)}$ and in some varieties of tomatoes ${ }^{(8)}$ and apples ${ }^{(9)}$. Dietary intake of stilbenes in Europe is between 2 and $3 \mathrm{mg} / \mathrm{d}$, and resveratrol and piceid represent about $50 \%$ of the total stilbene intake ${ }^{(10)}$

Resveratrol and piceid, after hydrolysis, are absorbed in the small intestine and extensively metabolised into glucuronides and sulphates. Unabsorbed resveratrol reaches the colon and is converted into dihydroresveratrol by the microbiota, which can also be absorbed, accounting for as much as $50 \%$ of an oral resveratrol dose ${ }^{(11)}$. Experiments with radiolabelled resveratrol have shown a high absorption of resveratrol in the gut, with more than $70 \%$ of the ingested radioactivity recovered in urine $^{(11)}$. To date, very little information is available on the bioavailability of other stilbenes such as piceatannol ${ }^{(12)}$ and viniferins ${ }^{(13)}$; although, to the best of our knowledge, they are not parent compounds of urinary resveratrol ${ }^{(14)}$.

Few studies assessing the ability of urinary resveratrol to serve as a biomarker of wine and resveratrol intake have been published. These studies have been conducted in Mediterranean countries where wine intake is high ${ }^{(15)}$. One interventional ${ }^{(16)}$ and two observational studies ${ }^{(17,18)}$ showed high correlations between habitual intake of wine or resveratrol and piceid and the concentration of resveratrol metabolites in spot urine samples. High correlations were also observed between dihydroresveratrol glucuronide and resveratrol concentrations measured in 24-h urine samples and acute intake of wine on the day of urine collection in a cross-sectional study in four European countries ${ }^{(19,20)}$.

In the present study, we aimed to investigate the correlation between acute and habitual dietary intakes of resveratrol and stilbenes, and their main food sources, and concentrations of urinary resveratrol in 24-h urine. First, our study assessed the usefulness of urinary resveratrol as a biomarker of resveratrol and wine consumption. Second, it evaluated the validity of using a dietary questionnaire (DQ) and the Phenol-Explorer food composition database ${ }^{(5)}$ to estimate the dietary resveratrol intake.

\section{Methods}

\section{Study population}

The European Prospective Investigation into Cancer and Nutrition (EPIC) study is a cohort study with over half a million participants of both sexes mostly recruited from the general population between 1992 and 2000 in twenty-three centres in ten European countries ${ }^{(21)}$. In a convenience subsample of 475 men and women, aged 50-61 years, a single 24-h dietary recall (24-HDR) and 24-h urine samples were collected on the same day between the years 1995 and $1999^{(20)}$. Approval for the study was obtained from the ethical review boards of the International Agency for Research on Cancer and from all participating institutions. All participants also provided written informed consent.

\section{Dietary and lifestyle information}

Short-term dietary data were collected using a single 24-HDR harmonised across countries (EPIC-Soft) ${ }^{(22)}$. The 24-HDR was administered in a face-to-face interview. Habitual dietary data were assessed at recruitment using a centre-specific quantitative DQ (France, Germany, Greece and Italy, except Naples) or a semi-quantitative FFQ (Naples) developed and validated in each country/centre ${ }^{(21,23)}$. The mean time interval between the DQ and the 24-HDR interview varied by country, from $1 \mathrm{~d}$ to 3 years later ${ }^{(24)}$. Dietary resveratrol and resveratrol 3-O-glucoside (piceid) intakes were estimated using Phenol-Explorer ${ }^{(5)}$, a comprehensive food composition database on polyphenols, as described in detail elsewhere ${ }^{(10)}$. Resveratrol aglycone equivalents were calculated as the sum of resveratrol and piceid contents after conversion of piceid to resveratrol equivalents on the basis of molecular weight. Total energy intake was estimated using the standardised EPIC Nutrient Database ${ }^{(25)}$.

Data on education, physical activity and smoking history were collected at baseline using questionnaires ${ }^{(24,26)}$. Data 
on age, body weight and height were self-reported by study participants during the 24-HDR interview.

\section{Samples and analytical method}

Urine samples over a $24-\mathrm{h}$ period were collected and stored at $-20^{\circ} \mathrm{C}$ using boric acid as a preservative. Completeness of the collection of 24-h urine samples was monitored using $p$-aminobenzoic acid (PABA). Samples with PABA recovery $<70$ and $>110 \%$ were excluded from this study ${ }^{(27)}$.

Resveratrol was analysed in 24-h urine samples using ultraperformance liquid chromatography-tandem $\mathrm{MS}^{(28)}$. In brief, urine samples were treated with a $\beta$-glucuronidase/sulfatase enzyme mixture and extracted twice with ethyl acetate; all phenolic groups in resveratrol were quantitatively dansylated using a differential isotope-labelling method and quantified using tandem MS. The limit of quantification (LOQ) for resveratrol was $0.02 \mu \mathrm{m}$. Inter-assay and intra-assay $\mathrm{CV}$ were $<10 \%$. Urinary excretion of resveratrol was expressed as $\mu \mathrm{mol} / 24-\mathrm{h}$. A small proportion of urinary excretion measurements $(n$ 46) did not meet predetermined analytical quality control criteria and were therefore excluded from statistical analysis.

\section{Statistical analyses}

Urinary resveratrol concentrations that fell below the LOQ were set to values corresponding to half the LOQ. Descriptive statistics (medians and 10th and 90th percentiles) including consumers and non-consumers were used for both urinary excretions and dietary intakes. Kruskal-Wallis tests were used to compare urinary resveratrol levels between groups of subjects with different baseline demographics and lifestyle characteristics. Spearman's rank correlations were used to assess the association between urinary resveratrol levels and dietary variables (stilbenes and stilbene-rich foods) estimated using DQ and 24-HDR. Partial Spearman's correlations $\left(R_{\text {partial }}^{2}\right)$ were computed to assess the contribution of dietary resveratrol intake (using DQ or 24-HDR) to urinary resveratrol levels while adjusting for other possible confounders: sex, BMI, age at recruitment, smoking status (never smoker, former smoker, current smoker) and total energy intake (measured in the DQ or in the $24-\mathrm{HDR}$, as appropriate). All statistical tests were two sided and the significance level was $P<0 \cdot 05$. All analyses were conducted using the Statistical Analysis Software, release 9.3 (SAS Institute Inc.).

\section{Results}

Of the 429 subjects with urinary resveratrol data, fifty-two participants had urinary resveratrol concentrations <LOQ. The median urinary excretions by centre, sex, age, BMI, smoking status and total energy intake are shown in Table 1. Excretion was higher in male subjects, in those aged 50-60 years and in those who were overweight (BMI $25-<30 \mathrm{~kg} / \mathrm{m}^{2}$ ) or had greater energy intake. The highest total urinary resveratrol concentrations were observed in Italy and France $(0 \cdot 09-0 \cdot 19 \mu \mathrm{mol} / 24 \mathrm{~h})$, whereas the lowest concentrations were observed in Greece $(0.04 \mu \mathrm{mol} / 24 \mathrm{~h})$. A similar pattern of
Table 1. Urinary resveratrol excretion $(\mu \mathrm{mol} / 24 \mathrm{~h})$ according to sociodemographic and lifestyle factors in the European Prospective Investigation into Cancer and Nutrition study (Medians and 10th and 90th percentiles (P10 and P90))

\begin{tabular}{|c|c|c|c|c|c|}
\hline Characteristics & $n$ & Median & P10 & P90 & $P$ \\
\hline Centre & & & & & $<0.001$ \\
\hline Paris (FRA) & 67 & 0.14 & 0.03 & 0.48 & \\
\hline Florence (ITA) & 45 & 0.17 & 0.03 & 0.84 & \\
\hline Varese (ITA) & 51 & 0.15 & 0.03 & 0.95 & \\
\hline Ragusa (ITA) & 17 & 0.19 & 0.04 & $1 \cdot 21$ & \\
\hline Turin (ITA) & 42 & 0.15 & 0.02 & 0.69 & \\
\hline Naples (ITA) & 20 & 0.09 & 0.03 & 0.84 & \\
\hline Greece & 56 & 0.04 & 0.02 & 0.10 & \\
\hline Heidelberg (GER) & 59 & 0.09 & 0.04 & 0.24 & \\
\hline Potsdam (GER) & 118 & 0.08 & 0.03 & 0.54 & \\
\hline Sex & & & & & 0.010 \\
\hline Men & 198 & 0.10 & 0.03 & 0.75 & \\
\hline Women & 277 & 0.09 & 0.02 & 0.41 & \\
\hline Age (years) & & & & & 0.061 \\
\hline$<50$ & 151 & 0.09 & 0.02 & 0.51 & \\
\hline $50-60$ & 201 & 0.11 & 0.03 & 0.60 & \\
\hline$>60$ & 123 & 0.08 & 0.02 & 0.48 & \\
\hline BMI $\left(\mathrm{kg} / \mathrm{m}^{2}\right)$ & & & & & 0.043 \\
\hline$<25$ & 207 & 0.09 & 0.02 & 0.65 & \\
\hline $25-<30$ & 186 & $0 \cdot 10$ & 0.03 & 0.53 & \\
\hline$\geq 30$ & 82 & 0.08 & 0.02 & 0.34 & \\
\hline Smoking status & & & & & 0.111 \\
\hline Never smoker & 241 & 0.09 & 0.02 & 0.48 & \\
\hline Former smoker & 129 & 0.10 & 0.03 & 0.71 & \\
\hline Current smoker & 92 & 0.07 & 0.02 & 0.42 & \\
\hline Unknown & 13 & 0.17 & 0.05 & 0.67 & \\
\hline Total energy intake $(\mathrm{kJ} / \mathrm{d})$ & & & & & 0.003 \\
\hline$<7448$ & 141 & 0.08 & 0.03 & 0.19 & \\
\hline 7448-9937 & 140 & 0.08 & 0.05 & 0.20 & \\
\hline$>9937$ & 148 & 0.11 & 0.06 & 0.31 & \\
\hline
\end{tabular}

FRA, France; ITA, Italy; GER, Germany.

habitual wine consumption by country was observed; the median intake ranged from $13 \mathrm{ml} / \mathrm{d}$ in Greece to $125 \mathrm{ml} / \mathrm{d}$ in Italian centres (data not tabulated).

Medians and 10th and 90th percentiles of the intake of resveratrol and resveratrol 3-O-glucoside and their food sources (e.g. wine, grapes, peanuts and red wine) are described in Table 2. Medians of the sum of resveratrol and piceid intake obtained using the 24-HDR were lower $(0 \cdot 10 \mathrm{mg} / \mathrm{d})$ than those obtained using the DQ $(0.42 \mathrm{mg} / \mathrm{d})$. As expected, a higher number of subjects with negligible intake of the sum of resveratrol and piceid was found using the 24-HDR $(n 82)$ than using the DQ $(n 0)$. Similar results were observed for wine, with medians varying between $0 \mathrm{~g} / \mathrm{d}$ (276 non-wine consumers) and $45 \mathrm{~g} / \mathrm{d}$ (seventy non-wine consumers) in the 24-HDR and in the DQ, respectively.

Spearman's correlation coefficients between urinary excretion and intakes of different dietary stilbenes were $>0.61$ and $>0.55$ as reported using 24-HDR and DQ (Table 3), respectively. The correlation between the sum of resveratrol and piceid intake and wine consumption was found to be 0.83 using 24-HDR and 0.95 using DQ. DQ data on red wine were collected only in Italy (except in Naples); therefore, data were available for 155 participants only. Correlations between red wine consumption and the sum of resveratrol and piceid intake were found to be lower in the 24-HDR $(r 0.71)$ than in the DQ ( $r$ 0.96). No significant correlations were found with either 
grape or peanuts. DQ data on peanut intake were collected only in Germany ( $n$ 177). The consumption of peanuts was negligible $(0 \mathrm{~g} / \mathrm{d}$ in the percentile 90 using both DQ and 24-HDR), and the number of peanut consumers was only

Table 2. Dietary intakes of resveratrol and piceid, their food sources and urinary excretion of resveratrol in the European Prospective Investigation into Cancer and Nutrition study ( $n$ 475)

(Medians and 10th and 90th percentiles (P10 and P90))

\begin{tabular}{|c|c|c|c|c|}
\hline Variables & $\begin{array}{l}\text { LOQ or non- } \\
\text { consumers }(n)\end{array}$ & Median & P10 & P90 \\
\hline \multicolumn{5}{|l|}{ Urine $24 \mathrm{~h}$} \\
\hline $\begin{array}{l}\text { Urinary resveratrol } \\
(\mu \mathrm{mol} / 24 \mathrm{~h})\end{array}$ & 52 & 0.09 & 0.02 & 0.54 \\
\hline \multicolumn{5}{|l|}{ 24-h dietary recall } \\
\hline Resveratrol (mg/d) & 84 & 0.04 & 0.00 & 0.81 \\
\hline $\begin{array}{l}\text { Resveratrol 3-O-glucoside } \\
(\mathrm{mg} / \mathrm{d})\end{array}$ & 163 & 0.02 & 0.00 & 1.66 \\
\hline $\begin{array}{l}\text { Resveratrol + resveratrol } \\
\text { 3-O-glucoside }(\mathrm{mg} / \mathrm{d})\end{array}$ & 82 & $0 \cdot 10$ & 0.00 & $2 \cdot 45$ \\
\hline Resveratrol aglycone $(\mathrm{mg} / \mathrm{d})$ & 82 & 0.07 & 0 & 1.74 \\
\hline Wine $(g / d)$ & 276 & 0.0 & 0.0 & $350 \cdot 0$ \\
\hline Grapes (g/d) & 458 & 0.0 & 0.0 & 0.0 \\
\hline Peanuts $(g / d)$ & 461 & 0.0 & 0.0 & 0.0 \\
\hline Red wine (g/d) & 354 & 0.0 & 0.0 & $260 \cdot 0$ \\
\hline \multicolumn{5}{|l|}{$\mathrm{DQ}$} \\
\hline Resveratrol (mg/d) & 0 & 0.17 & 0.03 & 0.70 \\
\hline $\begin{array}{l}\text { Resveratrol 3-O-glucoside } \\
(\mathrm{mg} / \mathrm{d})\end{array}$ & 0 & 0.24 & 0.02 & 1.57 \\
\hline $\begin{array}{l}\text { Resveratrol + resveratrol } \\
\text { 3-O-glucoside }(\mathrm{mg} / \mathrm{d})\end{array}$ & 0 & 0.42 & 0.06 & $2 \cdot 27$ \\
\hline Resveratrol aglycone $(\mathrm{mg} / \mathrm{d})$ & 0 & 0.33 & 0.05 & 1.61 \\
\hline Wine $(g / d)$ & 70 & 44.5 & 0.0 & 283.5 \\
\hline Grapes (g/d) & 31 & $7 \cdot 1$ & 0.3 & $33 \cdot 2$ \\
\hline Peanuts $(\mathrm{g} / \mathrm{d})^{\star}$ & 144 & 0.0 & 0.0 & 0.03 \\
\hline Red wine $(g / d) \dagger$ & 27 & 0.0 & $62 \cdot 5$ & 333.3 \\
\hline
\end{tabular}

$\mathrm{LOQ}$, limit of quantification; $\mathrm{DQ}$, dietary questionnaire.

* Peanut data only collected using the DQ in Germany ( $n$ 177).

† Red wine data only collected using the DQ in Italy, except Naples ( $n$ 155). fourteen of 475 subjects using the 24-HDR and thirty-three of 177 individuals using the DQ (Table 2).

The results of the multivariate-adjusted partial Spearman's correlations $\left(R_{\text {partial }}^{2}\right)$ between the intake of resveratrol and piceid, and their main food sources, and urinary resveratrol are shown in Table 4. $R_{\text {partial }}^{2}$ coefficients were similar $\left(R_{\text {partial }}^{2}\right.$ approximately 0.6 ) with resveratrol, piceid and wine using the 24-HDR. $R_{\text {partial }}^{2}$ coefficients were slightly lower $\left(R_{\text {partial }}^{2}\right.$ approximately 0.5 ) using data from the DQ. For red wine, the

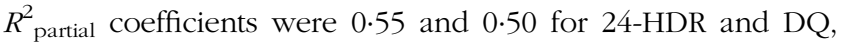
respectively.

\section{Discussion}

In the current study, resveratrol concentrations in 24-h urine were correlated with acute ( $R_{\text {partial }}^{2}$ approximately 0.6) and habitual $\left(R_{\text {partial }}^{2}\right.$ approximately 0.5$)$ dietary resveratrol, piceid and wine intake in a multicentre cohort of European adults. Habitual intake of the sum of resveratrol and piceid has previously been reported to correlate with concentrations of resveratrol metabolites ( $r$ 0.67), glucuronides and sulphates analysed in 24-h urine samples in a subsample of 783 men and women, aged 65 years and older, in the Italian InCHIANTI (Invecchiare in Chianti) study ${ }^{(18)}$. Higher correlations $(r$ 0.89) were observed between concentrations of resveratrol metabolites in morning urine samples and the habitual intake of resveratrol and piceid in a subsample of 1000 highcardiovascular-risk men and women in the Spanish PREDIMED (Prevención con Dieta Mediterránea) study ${ }^{(17)}$. The high correlation in the Spanish study could be due to the large proportion of red wine consumers ( $77 \%)$, whereas in our study the percentage of red wine consumers was much lower (37\%). In agreement with this hypothesis, we observed higher Spearman's correlations in countries with high wine consumption, such as Italy $(r 0 \cdot 62,38 \%$ non-wine consumers), than in Germany ( $r 0 \cdot 38,78 \%$ non-wine consumers). Therefore, urinary resveratrol concentration is a more

Table 3. Spearman's correlations between urinary resveratrol (RESV) concentrations and acute intakes of RESV, RESV 3-Oglucoside and their main food sources estimated with a single 24-h dietary recall and a dietary questionnaire in the European Prospective Investigation into Cancer and Nutrition study

\begin{tabular}{|c|c|c|c|c|c|c|c|c|}
\hline & $\begin{array}{l}\text { Urinary } \\
\text { RESV }\end{array}$ & RESV & $\begin{array}{c}\text { RESV } \\
\text { 3-O-glucoside }\end{array}$ & $\begin{array}{l}\text { RESV + RESV } \\
\text { 3-O-glucoside }\end{array}$ & $\begin{array}{l}\text { RESV } \\
\text { aglycone }\end{array}$ & Wine & Grape & Red wine \\
\hline \multicolumn{9}{|l|}{ 24-h dietary recall } \\
\hline Urinary RESV & & 0.61 & 0.63 & 0.64 & 0.63 & 0.61 & 0.09 & 0.59 \\
\hline RESV & & & 0.84 & 0.98 & 0.98 & 0.77 & 0.08 & 0.69 \\
\hline RESV 3-O-glucoside & & & & 0.92 & 0.90 & 0.85 & 0.07 & 0.73 \\
\hline RESV + RESV 3-O-glucoside & & & & & 0.99 & 0.83 & 0.07 & 0.71 \\
\hline RESV aglycone & & & & & & 0.92 & 0.07 & 0.71 \\
\hline Wine & & & & & & & -0.03 & 0.71 \\
\hline Grapes & & & & & & & & -0.04 \\
\hline Red wine & & & & & & & & \\
\hline \multicolumn{9}{|l|}{ Dietary questionnaire } \\
\hline Urinary RESV & & 0.57 & 0.55 & 0.57 & 0.58 & 0.56 & -0.01 & 0.61 \\
\hline RESV & & & 0.92 & 0.97 & 0.98 & 0.90 & 0.08 & 0.95 \\
\hline RESV 3-O-glucoside & & & & 0.98 & 0.97 & 0.96 & 0.07 & 0.95 \\
\hline RESV + RESV 3-O-glucoside & & & & & 0.99 & 0.95 & 0.07 & 0.96 \\
\hline RESV aglycone & & & & & & 0.94 & 0.07 & 0.96 \\
\hline Wine & & & & & & & -0.03 & 0.91 \\
\hline Grapes & & & & & & & & -0.03 \\
\hline Red wine* & & & & & & & & \\
\hline
\end{tabular}

* Red wine data only collected using the dietary questionnaire in Italy, except Naples ( $n$ 155) 
Table 4. $R_{\text {partial }}^{2}$ coefficients between dietary resveratrol and resveratrol 3-O-glucoside intake and urinary resveratrol concentrations in the European Prospective Investigation into Cancer and Nutrition study ( $n$ 429)*

\begin{tabular}{llc}
\hline Questionnaires & Dietary variable & Urinary resveratrol $(\mu \mathrm{mol} / 24 \mathrm{~h})$ \\
\hline 24-h dietary recall & Resveratrol $(\mathrm{mg} / \mathrm{d})$ & 0.57 \\
& Resveratrol 3-O-glucoside $(\mathrm{mg} / \mathrm{d})$ & 0.59 \\
& Resveratrol + resveratrol 3-O-glucoside $(\mathrm{mg} / \mathrm{d})$ & 0.59 \\
& Resveratrol aglycone $(\mathrm{mg} / \mathrm{d})$ & 0.59 \\
& Wine $(\mathrm{g} / \mathrm{d})$ & 0.59 \\
& Red wine $(\mathrm{g} / \mathrm{d})$ & 0.55 \\
Dietary questionnaire & Resveratrol $(\mathrm{mg} / \mathrm{d})$ & 0.51 \\
& Resveratrol 3-O-glucoside $(\mathrm{mg} / \mathrm{d})$ & 0.49 \\
& Resveratrol + resveratrol 3-O-glucoside $(\mathrm{mg} / \mathrm{d})$ & 0.51 \\
& Resveratrol aglycone $(\mathrm{mg} / \mathrm{d})$ & 0.51 \\
& Wine $(\mathrm{g} / \mathrm{d})$ & 0.51 \\
& Red wine $(\mathrm{g} / \mathrm{d})$ & 0.50 \\
\hline * &
\end{tabular}

suitable biomarker of resveratrol exposure in populations with elevated and regular intakes of resveratrol/wine than in those with low and occasional intakes.

Approximately $70 \%$ of dietary resveratrol and piceid consumed is recovered in urine as conjugates of resveratrol and dihydroresveratrol produced by the microbiota, which account for as much as $50 \%$ of an oral resveratrol dose ${ }^{(11)}$. Because of extensive metabolism in the gut and the liver, most of the resveratrol found in urine is glucuronidated and/or sulphated ${ }^{(29)}$, and only $1 \%$ of resveratrol absorbed is found in its free form in urine ${ }^{(11)}$. Therefore, in the analysis of urinary resveratrol, it is common practice to measure it after enzymatic hydrolysis to release resveratrol aglycone, as done here ${ }^{(28)}$. The current analytical method allowed us to accurately quantify urinary resveratrol using a proper standard, whereas, to date, standards for all conjugated metabolites of resveratrol are not commercially available. In addition, this method simultaneously analyses thirty-four urinary polyphenol aglycones, allowing us to potentially measure several nutritional biomarkers of polyphenol-rich foods at the same time ${ }^{(20)}$.

Assessment of resveratrol intake in populations represents a challenge for researchers primarily because of the variability and paucity of data on resveratrol content of some foods, although wine, particularly red wine, has been shown to be the main food source $(>92 \%)$ for total resveratrol and piceid intakes ${ }^{(4,10)}$. Resveratrol has been identified only in very few foods, mainly grapes, grape juice and wine. Chocolate, pistachios, peanuts and some varieties of berries ${ }^{(4,5)}$, beer $^{(7)}$, tomatoes ${ }^{(8)}$ and apples ${ }^{(9)}$ also contain resveratrol, but in very small concentrations, or they are rarely consumed (i.e. berries) (online Supplementary Table S1). Moreover, resveratrol content can vary greatly in the same type of fruit, depending on variety, degree of maturity at harvest, fungal pressure and climate ${ }^{(4,5)}$. Indeed, resveratrol content in red wines may vary up to 10 -fold mainly owing to grape variety, Pinot noir being the variety with the highest content ${ }^{(30)}$. The development of Phenol-Explorer in 2009 has facilitated the assessment of resveratrol intake in epidemiological studies ${ }^{(5)}$. However, to our knowledge, this is the first study correlating the estimation of dietary resveratrol intake using Phenol-Explorer with urinary resveratrol concentrations.

In the current study, similar correlations were observed between urinary resveratrol concentrations and dietary intake of either the sum of or individual resveratrol and piceid, or of resveratrol and piceid expressed as resveratrol aglycone equivalents. Therefore, urinary resveratrol excretion is a good predictor of dietary resveratrol and piceid. This is because wine, particularly red wine, is the main food source of resveratrol and piceid $(>92 \%)^{(4,10)}$. Likewise, in our study, urinary resveratrol level was also significantly correlated with wine consumption, especially with red wine intake, as previously described in other studies $^{(16,17)}$. Other non-resveratrol-related metabolites such as gallic acid ethyl ester, gallic acid ethyl ester sulphate, methylgallic acid sulphate, 4-O-methylgallic acid and hydroxytyrosol sulphate were also suggested as adequate biomarkers of red wine $^{(19,22)}$. Combinations of several metabolites could be better biomarkers of wine intake than individual compounds ${ }^{(31)}$.

Urinary excretion mainly takes place in the first $4 \mathrm{~h}$ after consumption ( $77 \%$ of total excretion), although resveratrol metabolites remain in urine for at least $24 \mathrm{~h}$ after intake ${ }^{(29,32)}$. Therefore, correlations between urinary and dietary resveratrol were found to be higher when using 24-HDR ( $R_{\text {partial }}^{2}$ approximately $0 \cdot 6$ ), a dietary assessment tool for short-term and acute dietary intake, than when using DQ ( $R_{\text {partial }}^{2}$ approximately 0.5$)$, a tool for long-term and habitual dietary intake. Similar findings with other polyphenols comparing short- with long-term dietary exposures have been reviewed previously ${ }^{(33)}$. Despite this, partial correlations using DQ were still significant. This is due to the fact that wine was consumed on a regular basis by European adults in the 1990s, especially in wine-producing countries such as France, Italy and Spain. For example, the number of red wine consumers was 121 of 475 and 128 of 155 subjects (data collected only in some Italian centres) on using the 24-HDR and DQ, respectively. Moreover, using urinary resveratrol metabolites as biomarkers of wine consumption, Zamora-Ros et al. were able to differentiate non-wine consumers from sporadic consumers (one to three glasses of wine per week) who had consumed their last glasses 3-5 d before urine collection ${ }^{(17)}$. Overall, urinary resveratrol concentrations are adequate biomarkers of short-term and habitual dietary resveratrol intake in countries where wine is regularly consumed.

Our results may be influenced by measurement errors in the 24-HDR and DQ, which may attenuate our findings. However, the 24-HDR was collected using standardised software (EPIC-Soft) and administered in a face-to-face interview ${ }^{(22)}$. Furthermore, DQ were country-specific and previously validated for some resveratrol-rich foods such as wine ${ }^{(23)}$. Second, estimation of dietary resveratrol 
intake may be limited by the incompleteness of Phenol-Explorer, although it is currently the most comprehensive database on polyphenols including stilbenes ${ }^{(5)}$. Finally, the results might also be influenced by laboratory measurement error, although our analytical methodology has fulfilled all criteria to be validated ${ }^{(28)}$

In conclusion, urinary excretion of resveratrol was significantly and positively associated with resveratrol, stilbene and wine intakes estimated by 24-HDR and DQ. The correlation coefficients demonstrate that urinary resveratrol is an adequate biomarker of both acute and habitual resveratrol and wine intake in this population. Moreover, our results support the validity of our methodology, 24-HDR, DQ and the food composition database for estimating resveratrol intake. The findings of this study lay the groundwork for future studies to identify the role of resveratrol in the risk for chronic diseases.

\section{Acknowledgements}

The authors thank Mr Bertrand Hémon for his valuable help with the EPIC database.

This work was supported by the Institut National du Cancer, Paris (INCa grant 2011-105) and the Wereld Kanker Onderzoek Fonds (WCRF NL 2012/604). The national cohorts are supported by the French National Cancer Institute (L'Institut National du Cancer; grant 2009-139); Ligue contre le Cancer, Institut Gustave Roussy, Mutuelle Générale de l'Education Nationale, Institut National de la Santé et de la Recherche Médicale (France); German Cancer Aid; German Cancer Research Center (DKFZ); German Federal Ministry of Education and Research (Germany); the Hellenic Health Foundation (Greece); Italian Association for Research on Cancer; Compagnia San Paolo, (Italy); Agència de Gestió d'Ajuts Universitaris i de Recerca (AGAUR) - Generalitat de Catalunya (exp. 2014 SGR 726), The Health Research Funds (RD12/0036/0018) (Spain). R. Z.-R. would like to thank the 'Miguel Servet' program (CP15/00100) from the Institute of Health Carlos III and European Social Fund.

R. Z.-R. and A. S. designed the research; R. Z.-R. wrote the manuscript; R. Z.-R. and J. A. R. analysed the data; J. A. R., D. A., A. S., P. F., M.-C. B.-R., F. R. M., A. A., T. K., V. K., H. B., S. K., A. T., P. L., C. L. V., D. P., P. C., S. P., R. T., F. R., H. N., H. F., I. R. reviewed and edited the manuscript; D. A. performed the laboratory analyses; A. S., P. F., M.-C. B.-R., F. R. M., A. A., T. K., V. K., H. B., S. K., A. T., P. L., C. L. V., D. P., P. C., S. P., R. T., F. R., H. N., H. F., I. R. contributed to the discussion. All authors have read and approved the final manuscript.

The authors declare that there are no conflicts of interest.

\section{Supplementary material}

For supplementary material/s referred to in this article, please visit https://doi.org/10.1017/S0007114517001465

\section{References}

1. Baur JA \& Sinclair DA (2006) Therapeutic potential of resveratrol: the in vivo evidence. Nat Rev Drug Discov 5, 493-506.
2. Tome-Carneiro J, Larrosa M, Gonzalez-Sarrias A, et al. (2013) Resveratrol and clinical trials: the crossroad from in vitro studies to human evidence. Curr Pharm Des 19, 6064-6093.

3. Rabassa M, Zamora-Ros R, Urpi-Sarda M, et al. (2015) Resveratrol metabolite profiling in clinical nutrition research-from diet to uncovering disease risk biomarkers: epidemiological evidence. Ann N Y Acad Sci 1348, 107-115.

4. Zamora-Ros R, Andres-Lacueva C, Lamuela-Raventos RM, et al. (2008) Concentrations of resveratrol and derivatives in foods and estimation of dietary intake in a Spanish population: European Prospective Investigation into Cancer and Nutrition (EPIC)-Spain cohort. Br J Nutr 100, 188-196.

5. Neveu V, Perez-Jimenez J, Vos F, et al. (2010) PhenolExplorer: an online comprehensive database on polyphenol contents in foods. Database (Oxford) 2010, bap024.

6. Hurst WJ, Glinski JA, Miller KB, et al. (2008) Survey of the trans-resveratrol and trans-piceid content of cocoa-containing and chocolate products. J Agric Food Chem 56, 8374-8378.

7. Chiva-Blanch G, Urpi-Sarda M, Rotches-Ribalta M, et al. (2011) Determination of resveratrol and piceid in beer matrices by solid-phase extraction and liquid chromatography-tandem mass spectrometry. J Chromatogr A 1218, 698-705.

8. Ragab AS, Van FJ, Jankowski B, et al. (2006) Detection and quantitation of resveratrol in tomato fruit (Lycopersicon esculentum Mill.). J Agric Food Chem 54, 7175-7179.

9. Farneti B, Masuero D, Costa F, et al. (2015) Is there room for improving the nutraceutical composition of apple? J Agric Food Chem 63, 2750-2759.

10. Zamora-Ros R, Knaze V, Rothwell JA, et al. (2015) Dietary polyphenol intake in Europe: the European Prospective Investigation into Cancer and Nutrition (EPIC) study. Eur J Nutr 55, 1359-1375.

11. Walle T, Hsieh F, DeLegge MH, et al. (2004) High absorption but very low bioavailability of oral resveratrol in humans. Drug Metab Dispos 32, 1377-1382.

12. Lin HS, Tringali C, Spatafora C, et al. (2010) A simple and sensitive HPLC-UV method for the quantification of piceatannol analog trans-3,5,3',4'-tetramethoxystilbene in rat plasma and its application for a pre-clinical pharmacokinetic study. J Pharm Biomed Anal 51, 679-684.

13. Willenberg I, Michael M, Wonik J, et al. (2015) Investigation of the absorption of resveratrol oligomers in the Caco-2 cellular model of intestinal absorption. Food Chem 167, 245-250.

14. Rothwell JA, Urpi-Sarda M, Boto-Ordonez M, et al. (2012) Phenol-Explorer 2.0: a major update of the Phenol-Explorer database integrating data on polyphenol metabolism and pharmacokinetics in humans and experimental animals. Database (Oxford) 2012, bas031.

15. Sieri S, Agudo A, Kesse E, et al. (2002) Patterns of alcohol consumption in 10 European countries participating in the European Prospective Investigation into Cancer and Nutrition (EPIC) project. Public Health Nutr 5, 1287-1296.

16. Zamora-Ros R, Urpi-Sarda M, Lamuela-Raventos RM, et al. (2006) Diagnostic performance of urinary resveratrol metabolites as a biomarker of moderate wine consumption. Clin Chem 52, 1373-1380.

17. Zamora-Ros R, Urpi-Sarda M, Lamuela-Raventos RM, et al. (2009) Resveratrol metabolites in urine as a biomarker of wine intake in free-living subjects: the PREDIMED Study. Free Radic Biol Med 46, 1562-1566.

18. Semba RD, Ferrucci L, Bartali B, et al. (2014) Resveratrol levels and all-cause mortality in older community-dwelling adults. JAMA Intern Med 174, 1077-1084

19. Edmands WM, Ferrari P, Rothwell JA, et al. (2015) Polyphenol metabolome in human urine and its association with intake of 
polyphenol-rich foods across European countries. Am J Clin Nutr 102, 905-913.

20. Zamora-Ros R, Achaintre D, Rothwell JA, et al. (2016) Urinary excretions of 34 dietary polyphenols and their associations with lifestyle factors in the EPIC cohort study. Sci Rep 6, 26905.

21. Riboli E \& Kaaks R (1997) The EPIC Project: rationale and study design. European Prospective Investigation into Cancer and Nutrition. Int J Epidemiol 26, Suppl. 1, S6-S14.

22. Slimani N, Ferrari P, Ocke M, et al. (2000) Standardization of the 24-hour diet recall calibration method used in the European Prospective Investigation into Cancer and Nutrition (EPIC): general concepts and preliminary results. Eur J Clin Nutr 54, 900-917.

23. Margetts BM \& Pietinen P (1997) European Prospective Investigation into Cancer and Nutrition: validity studies on dietary assessment methods. Int J Epidemiol 26, Suppl. 1, S1-S5.

24. Slimani N, Kaaks R, Ferrari P, et al. (2002) European Prospective Investigation into Cancer and Nutrition (EPIC) calibration study: rationale, design and population characteristics. Public Health Nutr 5, 1125-1145.

25. Slimani N, Deharveng G, Unwin I, et al. (2007) The EPIC nutrient database project (ENDB): a first attempt to standardize nutrient databases across the 10 European countries participating in the EPIC study. Eur J Clin Nutr 61, 1037-1056.

26. Wareham NJ, Jakes RW, Rennie KL, et al. (2003) Validity and repeatability of a simple index derived from the short physical activity questionnaire used in the European Prospective Investigation into Cancer and Nutrition (EPIC) study. Public Health Nutr 6, 407-413.
27. Slimani N, Bingham S, Runswick S, et al. (2003) Group level validation of protein intakes estimated by 24 -hour diet recall and dietary questionnaires against 24-hour urinary nitrogen in the European Prospective Investigation into Cancer and Nutrition (EPIC) calibration study. Cancer Epidemiol Biomarkers Prev 12, 784-795.

28. Achaintre D, Bulete A, Cren-Olive C, et al. (2016) Differential isotope labelling of 38 dietary polyphenols and their quantification in urine by liquid chromatography/electrospray ionization tandem mass spectrometry. Anal Chem 88, 2637-2644.

29. Rotches-Ribalta M, Andres-Lacueva C, Estruch R, et al. (2012) Pharmacokinetics of resveratrol metabolic profile in healthy humans after moderate consumption of red wine and grape extract tablets. Pharmacol Res 66, 375-382.

30. Lamuela-Raventós R, Romero-Pérez A, Waterhouse A, et al. (1995) Direct HPLC analysis of cis- and trans-resveratrol and piceic isomers in Spanish red Vitis vinifera wines. J Agric Food Chem 43, 281-283.

31. Noh H, Freisling H, Assi N, et al. (2017) Profiles of urinary polypenol metabolites associated with polyphenol-rich food intake in the European Prospective Investigation into Cancer and Nutrition (EPIC) study. Nutrients (In the Press).

32. Boocock DJ, Faust GE, Patel KR, et al. (2007) Phase I dose escalation pharmacokinetic study in healthy volunteers of resveratrol, a potential cancer chemopreventive agent. Cancer Epidemiol Biomarkers Prev 16, 1246-1252.

33. Zamora-Ros R, Touillaud M, Rothwell JA, et al. (2014) Measuring exposure to the polyphenol metabolome in observational epidemiologic studies: current tools and applications and their limits. Am J Clin Nutr 100, 11-26. 